Proceedings of the 1st international conference Economic and Business Trends Shaping the Future $\mid 2020$

\title{
AUDIT PROFESSION IN THE SHADOW OF THE COVID -19 - A PERCEPTION OF AUDITORS IN REPUBLIC OF NORTH MACEDONIA
}

\author{
Zorica Bozhinovska Lazarevska, Ph.D. \\ Faculty of Economics - Skopje, Ss. Cyril and Methodius University \\ zorica.bozinovska-lazarevska@eccf.ukim.edu.mk \\ Marina Trpeska, Ph.D. \\ Faculty of Economics - Skopje, Ss. Cyril and Methodius University \\ marina.serafimoska@eccf.ukim.edu.mk \\ Atanasko Atanasovski, Ph.D. \\ Faculty of Economics - Skopje, Ss. Cyril and Methodius University \\ atanasko.atanasovski@eccf.ukim.edu.mk \\ Ivan Dionisijev, M.Sc. \\ Faculty of Economics - Skopje, Ss. Cyril and Methodius University \\ ivan.d@eccf.ukim.edu.mk
}

\begin{abstract}
The virtual practice has posed serious challenges and opened many dilemmas for the auditors. How to obtain sufficient and appropriate audit evidence in the absence of communication with the client, to assess the risks of material misstatement, to recognize the symptoms of fraud, to check subsequent events, to assess the ability for 'going concern', to exercise the required level of professional skepticism, and how to write an audit report in which the auditor will offer a reasonable assurance to users of the opinion expressed. Health experts warn that even in the postCOVID-19 period, the social distance will be an integral part of our lives. In that regard, IFAC also points out the need to prepare auditors for work in the so-called "the new normal". The paper researches how the COVID-19 pandemic affects the audit profession in the Republic of North Macedonia. For that purpose, our research is based on a survey distributed to the certified auditors, members of IORRM (Institute of Certified Auditors of the Republic of North Macedonia). From the questions asked, we have concluded whether and how much the pandemic has changed the approach of auditors in conducting audit engagements, how auditors have managed to amortize the initial shock, whether they have prepared a strategy for virtual practice in the environment of the so-called "new normal" and what are the biggest challenges they expect to face in the post-COVID world. In general, it can be concluded that all challenges posed by the pandemic will be more easily overcome by joint and smart action of certified auditors and related associations and regulatory bodies, in order to find alternative ways of acting in a radically changed environment under the influence of the COVID-19 pandemic. In addition to analyzing data obtained from the survey, documents related to the impact of COVID-19 pandemic worldwide are used, as well as other literature and scientific papers on how the audit profession responds to the COVID crisis.
\end{abstract}

Keywords: Pandemic, COVID-19, Audit Profession, Auditors

\section{JEL classification: M40, M42}

http://doi.org/10.47063/EBTSF.2020.0006

http://hdl.handle.net/20.500.12188/9683 


\section{INTRODUCTION}

Throughout history, the disease outbreaks have devastated humankind, sometimes changing the course of history, and at times even ending entire civilizations. Every crisis is different and they can disrupt our home life, our places of work, and our mindset, with repercussions for internal controls and processes, personal and professional behavior, and decisions made in the face of uncertainty and incomplete information. The International Federation of Accountants (IFAC, 2020) points out that such challenges impact the important services, professional accountants provide and can shake the foundation upon which relevant, reliable, and high-quality information - relied on by boards and the leadership of reporting entities, government/regulatory policymakers, investors, and other stakeholders - is based. When these challenges emerge and the importance of high-quality information is heightened, the expertise, trust, and judgment of the accountancy profession are tested and must truly shine. Every crisis teaches us something new about how best to prepare for, mitigate, or even try to prevent the next event (IFAC, 2020).

COVID-19 Pandemic in 2020 is proof that the world is not immune to new viruses and diseases, which can be significantly fatal in many areas of society (GHRF Commission, 2016). The unprecedented current situation caused by COVID-19, has disrupted most professions across the globe with accounting and auditing being no exception. Mandatory lockdown measures were imposed by governments to control the spread of the virus, with individuals having to work from home where possible. For auditors, this means they can no longer travel to audited entity premises, nor even to their own offices and that their audits will have to be completed remotely. The Association of Chartered Certified Accountants (ACCA, 2020) indicates that there is a positive side to this significant challenge for auditors, and that is that the audit profession was already on a journey to becoming more digital, and the investment in digital capability has allowed many firms and practitioners to adapt to the new circumstances relatively quicker than other industries (ACCA, 2020). All professional accountants need to continue to exercise high levels of diligence, integrity, and professional judgment. They, whether serving as members of a board or audit committee, organizational leaders, preparers, or auditors, are critical to the financial reporting ecosystem (IFAC, 2020).

Metaphorically, Brachio (2020) indicates that at a big-picture level, the most powerful learning to take away from the COVID-19 crisis is that there is always a potential "black swan" event around the corner - that is, an unpredictable event that has a severe impact. And with today's global connectivity, that event could again impact on the entire world. The risks associated with "black swan" events tend to be so big and so rare that many businesses do not contemplate them, much less plan for them. The auditors need to have a mindset where they are always prepared to think the unthinkable - because the "black swan" will show up (Brachio, 2020).

This paper highlights some of the practical challenges that auditors are now faced with, in light of COVID-19, and highlights some of the key considerations for auditors by referring to the relevant International Auditing Standards.

In this paper, we discuss the impact of the novel Coronavirus (COVID-19) outbreak on the auditing profession in the Republic of North Macedonia which not only includes writing an audit report in which the auditor will offer a reasonable assurance to users of the opinion expressed, but also obtaining sufficient and appropriate audit evidence in the absence of communication with the client, assessing the risks of material misstatement, recognizing the symptoms of fraud, checking subsequent events, assessing the ability for 'going concern', exercising the required level of professional skepticism.

We believe that this is the first study about the impact of the outbreak of COVID-19 on the auditing profession from the viewpoint of the Certified Auditors in the Republic of North Macedonia. 


\section{LITERATURE REVIEW}

In the past and today, the impact of pandemics on the audit profession has not been well researched, given the fact that pandemics located worldwide with major consequences and changes in people's lives, such as COVID-19 Pandemic, are not common.

The need for the professional accountants in the society is indicated by IFAC in the recent issue of 'IFAC Point of View', that defines them as an essential component of a sustainable and resilient global economy, guided by their fundamental ethical responsibility to act in the public interest. Maintaining trust and confidence during a crisis, such as the pandemics, is the fastest path to recovery after a crisis (IFAC, 2020). Dohrer \& Mayes (2020) point out that many auditors have begun to turn their next group of audits: clients with the 2020 fiscal year ends. The World Health Organization declared a public health emergency on Jan. 30, 2020, meaning many of these clients will have been affected by the COVID-19 Pandemic during the period under audit. Auditing these clients will carry unique challenges, and certain areas may present heightened risks of material misstatement for the audit (Dohrer \& Mayes, 2020).

According to the International Organization of Securities Commissions (IOSCO, 2020) in their Statement on Importance of Disclosure about COVID-19, it is observed that the COVID-19 presents a veritable "perfect storm" for fraud risk, and auditors should be on high alert. As it is stated in the IOSCO's statement, the investors and other stakeholders need timely and high-quality financial information complete with transparent and entity-specific disclosures, including information about the impact of COVID-19 on the issuer's operating performance, financial position, liquidity, and prospects. In terms of annual financial reporting, a critical part of the ecosystem that provides reliable, high-quality financial information to investors is the high-quality audits conducted by an independent auditor. Further, IOSCO (2020) reminds the auditors of their responsibilities to report on Key Audit Matters, including a description of how the auditor addressed these matters. Additionally, there may be circumstances for which the auditor concludes that the audit report should contain an emphasis of matter paragraph or a material uncertainty relating to going concern paragraph. Auditors should modify their reports when the standards require to do so (e.g., if there is a material uncertainty about the entity's ability to continue as going concern and this uncertainty has not been adequately indicated in the notes or the management commentary). In this regard, IOSCO reminds external auditors of their responsibility to communicate with management and audit committees and to evaluate the adequacy and transparency of disclosures provided in the financial statements (IOSCO, 2020).

\section{OBJECTIVE OF THE RESEARCH AND A QUESTIONNAIRE DEVELOPMENT}

As it is mentioned earlier in this paper, the audit profession in the first half of 2020 was faced with unknown circumstances due to COVID-19. As the pandemic persists later in the year and still is ongoing with immense impact on the whole economy affecting almost all companies, the need for audited financial statement gain on its popularity. On the one hand, we have companies that are negatively affected and are faced with serious challenges to survive in during this pandemic period working with reduced demand, reduced human capital, and reduced resources overall, and on the other hand companies with increased demand but also with reduced human capital due to saving human lives. In the situation when most of the companies have fully or partially moved to work from home relying on technologies internal controls are put on a serious test. All of these circumstances only complicate the work of auditors requiring them to be adaptable to the new situation and new working environment for themselves and their clients without endangering the quality of audits. 
For the purpose of this research, a survey was conducted in July and August 2020, by an online questionnaire that consists of all aspects of the audit process, change working environment, need for changes into the law and regulation, implications on the audit reports for both 2019 and 2020, as well as the expectations of the auditors for the profession in the post-COVID period. Therefore, the research is based on five main objectives:

- To analyze how auditors' response to the new unpredictable situation caring for their health and the quality of their work.

- What changes they do, if any, in the audit process on the key audit elements?

- Does the pandemic has implications on the audit reports for the financial statements for 2019 and would they expect implication on the audit reports for 2020.

- To see their opinion or requirements for changes in the regulation.

- What are their expectations for the future of the audit profession in the post-COVID period?

Certified auditors are coded and categorized according to their current practical experience and place of work as auditors in "Big Four", other international audit networks, local audit firms, certified auditor - sole proprietor, and other (banks, insurance companies, consultancy firms, university, public companies). The survey was intended to take no more than 10 minutes. Most of the questions (except introductory and preliminary questions) were answered on a Likert scale from 1 to 5 where 1 is totally disagree and 5 is totally agree. Introductory and preliminary questions include where the certified auditors work ( 1 and 2 questions) and which services they are providing (3 question) in order to research the differences in views, for example, certified auditors in audit practice and certified auditors engaged in other activities (internal audit, bookkeeping, education etc.). These questions are given in Appendix A (Table A-1) and in further analysis, for the process simplification, only the number of the question is given.

Before starting with any research analysis of the results, reliability analysis was carried out. Cronbach's alfa coefficient was calculated in order to measure the internal consistency and reliability of the questionnaire. Its result is 0.872 which implies high internal consistency and all items appeared to be worthy of retention.

\section{RESEARCH APPROACH TO ANALYZING RESPONSES AND RESULTS}

As it is stated earlier in the study, the main purpose of the study is to provide empirical evidence on certified auditors' perceptions about working in a pandemic environment. The survey was webbased and it was sent to 120 certified auditors in the Republic of North Macedonia. Participants received an invitation email that provided a link to the survey. After a second reminding by email 62 responses are received, or a 51.7 response rate.

As it is mentioned earlier in this paper, most of the analysis are made on structured sample into the five groups - auditors from "Big Four" (10), auditors from other international audit companies (18), auditors from local audit companies (13), certified auditor - sole proprietor (8) and other (13). The group other mostly consists of certified auditors working as internal auditors, professors, or quality controllers.

It should be emphasized even in the very beginning of this analysis that all auditors employed within "Big Four" that audit quality (Q22) should not be sacrificed in the circumstances of working from home, restricted communication with the clients, closing the offices of the clients subject to audit and lockdowns (Table 1). Also, the other groups present high means, all above 4.4, and the conclusion based on ANOVA is that there is no difference between the groups related with their 
attitude for the audit quality. Related to the quality of the audit profession during the COVID-19 pandemic (Q24) auditors with a mean of 4.45 agree that the risks imposed by the pandemic in the aspect of a true and objective presentation of financial statements emphasize the importance of quality accounting and auditing profession. There is no difference within the groups upon this question.

Auditors also answered with high certainty question 5 (Mean 4) that their companies were ready to apply the IFAC recommendations to work from home in order to save the health of the employees. This is an indication for the flexibility of the audit profession and its readiness to perform the audit in the changed environment without sacrificing the audit quality. However, there is a significant difference within the groups, especially between international audit companies (Big Four and other international audit companies) and local audit companies and certified auditor sole proprietor.

Regarding the financial statements and expectations of the auditors and stakeholders from the audit report, auditors believe that world pandemic would slightly increase the expectations of the investors and other stakeholders for presenting quality financial statements (Q21) and will not increase the expectation gap between auditors and audit reports' users (Q23). Also, auditors do not believe that there will be increased demand for changed range of services offered by audit companies (Q25).

However, the majority of questions are dedicated to the audit process, and the impact of the world pandemic over it. Surprisingly, auditors are consent that COVID-19 pandemic will not influence the audit process in big part. They even have said that annual audit plan (Q7) will be subject only on minor changes. Uncertainties imposed by COVID-19 do not have big impact on auditors' professional judgment in the segment of reassessment of preliminary assigned levels of materiality and audit risk (Q8), as well as do not have big impact on the way of collecting and documenting audit evidence (Q9). This is the case also with the auditors' perception of the accounting estimations (Q10). Interesting is the fact that although the whole national economies are faced with difficulties and lowering levels of growth, where many companies are on the threshold of survival, auditors do not expect big changes of their clients' portfolio (Q11). For all these variables according to ANOVA there is no significant difference between the groups. The question related with the increasing the level of the professional skepticism due to the increased risk of material misstatements in the financial statements (Q12) shows that auditors have slightly increased professional skepticism and what is more interesting there is significant difference between the groups. In fact, auditors work in "Big Four" companies increase the level of professional skepticism more significantly than the other auditors.

The next three questions (Q13, Q14 and Q15) are related with the internal controls and frauds. There are no significant differences between groups in all three questions. It is interesting that auditors, again do believe that there is some non-significant change in the internal controls and their functioning, as well as with the elements of fraud triangle and even with the identification of the "red flags". Auditors believe that working from home and lockdowns do not contribute in significant increasing of frauds.

Regarding the audit reports for the audited financial statements for the year ended on 31 December 2020, auditors do not believe that there will be increased number of modified opinions (Q18), but do believe that there will be increased number of paragraphs with emphasis of matters (Q19).

Regarding the compliance with law and regulation, standards and ethics, auditors do not expect noncompliance with the regulation and standards (Q16) and also do not expect big compromises of the basic ethical principles from the International Code of Ethics of IFAC. For this question there is a significant difference between the groups. 
At the end, having in mind the whole constellation of working environment beside the requirements for retaining and increasing the quality of audit work, auditors expect decreasing of their revenues for the period of the world pandemic (Q16).

For the post-COVID period auditors, as it is expected in all other professions, expect changing of the skills and knowledge required for the audit that should be included in the programs for professional certification of auditors (Q33).

Table 1. Case summaries

\begin{tabular}{|c|c|c|c|c|c|c|c|c|c|c|c|c|}
\hline & & 4 & 5. & 7. & 8. & 9. & 10. & 11. & 12. & 13. & 14. & 15. \\
\hline \multirow[t]{6}{*}{ Big Four } & $\mathrm{N}$ & 10 & 8 & 8 & 8 & 8 & 8 & 8 & 8 & 8 & 8 & 8 \\
\hline & Mean & 3.80 & 4.75 & 2.75 & 2.63 & 3.13 & 2.88 & 2.38 & 4.25 & 3.75 & 2.88 & 3.00 \\
\hline & Std. Deviation & 1.033 & .463 & .886 & 1.302 & 1.126 & 1.356 & 1.188 & .707 & .886 & 1.356 & 1.309 \\
\hline & Variance & 1.067 & .214 & .786 & 1.696 & 1.268 & 1.839 & 1.411 & .500 & .786 & 1.839 & 1.714 \\
\hline & Sum & 38 & 38 & 22 & 21 & 25 & 23 & 19 & 34 & 30 & 23 & 24 \\
\hline & $\%$ of Total $\mathrm{N}$ & $16.1 \%$ & $14.8 \%$ & $15.1 \%$ & $15.1 \%$ & $14.8 \%$ & $15.1 \%$ & $16.0 \%$ & $15.4 \%$ & $14.8 \%$ & $14.5 \%$ & $14.5 \%$ \\
\hline \multirow{6}{*}{\begin{tabular}{|l|} 
Other \\
Internatio \\
nal audit \\
network
\end{tabular}} & $\mathrm{N}$ & 18 & 18 & 17 & 18 & 18 & 18 & 18 & 18 & 18 & 18 & 18 \\
\hline & Mean & 3.22 & 4.06 & 3.06 & 2.17 & 2.94 & 2.33 & 2.28 & 2.83 & 2.72 & 2.61 & 2.72 \\
\hline & Std. Deviation & 1.060 & .639 & 1.029 & .618 & .998 & .907 & 1.179 & 1.098 & 1.074 & .850 & 1.074 \\
\hline & Variance & 1.124 & .408 & \begin{tabular}{|l|}
1.059 \\
\end{tabular} & .382 & .997 & .824 & 1.389 & 1.206 & 1.154 & .722 & 1.154 \\
\hline & Sum & 58 & 73 & 52 & 39 & 53 & 42 & 41 & 51 & 49 & 47 & 49 \\
\hline & $\%$ of Total $\mathrm{N}$ & $29.0 \%$ & $33.3 \%$ & $32.1 \%$ & $34.0 \%$ & $33.3 \%$ & $34.0 \%$ & $36.0 \%$ & $34.6 \%$ & $33.3 \%$ & $32.7 \%$ & $32.7 \%$ \\
\hline \multirow{6}{*}{\begin{tabular}{|l|} 
Local \\
audit \\
companie \\
$\mathrm{S}$
\end{tabular}} & $\mathrm{N}$ & 13 & 13 & 13 & 13 & 13 & 13 & 13 & 13 & 13 & 13 & 13 \\
\hline & Mean & 3.15 & 3.77 & 2.62 & 2.31 & 3.00 & 2.31 & 1.92 & 2.69 & 2.77 & 2.54 & 2.85 \\
\hline & Std. Deviation & 1.068 & .725 & .870 & .855 & 1.000 & .947 & .641 & .947 & .832 & .877 & 1.144 \\
\hline & Variance & 1.141 & .526 & .756 & .731 & 1.000 & .897 & .410 & .897 & .692 & .769 & 1.308 \\
\hline & Sum & 41 & 49 & 34 & 30 & 39 & 30 & 25 & 35 & 36 & 33 & 37 \\
\hline & $\%$ of Total $\mathrm{N}$ & $21.0 \%$ & $24.1 \%$ & $24.5 \%$ & $24.5 \%$ & $24.1 \%$ & $24.5 \%$ & $26.0 \%$ & $25.0 \%$ & $24.1 \%$ & $23.6 \%$ & $23.6 \%$ \\
\hline \multirow{6}{*}{\begin{tabular}{|l|} 
Certified \\
auditor - \\
sole \\
proprietor
\end{tabular}} & $\mathrm{N}$ & 8 & 8 & 8 & 8 & 8 & 8 & 8 & 8 & 8 & 8 & 8 \\
\hline & Mean & 3.63 & 3.75 & 3.25 & 2.38 & 3.38 & 2.88 & 2.25 & 3.38 & 3.25 & 2.88 & 2.75 \\
\hline & Std. Deviation & 1.188 & .463 & \begin{tabular}{|l|}
1.389 \\
\end{tabular} & .916 & 1.061 & 1.126 & .707 & $\mid 1.188$ & .886 & 1.356 & 1.165 \\
\hline & Variance & 1.411 & .214 & 1.929 & .839 & 1.125 & 1.268 & .500 & 1.411 & .786 & 1.839 & 1.357 \\
\hline & Sum & 29 & 30 & 26 & 19 & 27 & 23 & 18 & 27 & 26 & 23 & 22 \\
\hline & $\%$ of Total $\mathrm{N}$ & $12.9 \%$ & $14.8 \%$ & $15.1 \%$ & $15.1 \%$ & $14.8 \%$ & $15.1 \%$ & $16.0 \%$ & $15.4 \%$ & $14.8 \%$ & $14.5 \%$ & $14.5 \%$ \\
\hline \multirow[t]{6}{*}{ Other } & $\mathrm{N}$ & 13 & 7 & 7 & 6 & 7 & 6 & 3 & 5 & 7 & 8 & 8 \\
\hline & Mean & 3.15 & 3.71 & 3.14 & 2.33 & 2.86 & 2.33 & 3.00 & 2.40 & 2.86 & 2.88 & 3.00 \\
\hline & Std. Deviation & 1.068 & .756 & 1.069 & 1.033 & 1.215 & 1.033 & 1.732 & 1.140 & 1.069 & .991 & .756 \\
\hline & Variance & 1.141 & .571 & 1.143 & 1.067 & 1.476 & 1.067 & 3.000 & \begin{tabular}{|l|l|}
1.300 \\
\end{tabular} & 1.143 & .982 & .571 \\
\hline & Sum & 41 & 26 & 22 & 14 & 20 & 14 & 9 & 12 & 20 & 23 & 24 \\
\hline & $\%$ of Total $\mathrm{N}$ & $21.0 \%$ & $13.0 \%$ & $13.2 \%$ & $11.3 \%$ & $13.0 \%$ & $11.3 \%$ & $6.0 \%$ & $9.6 \%$ & $13.0 \%$ & $14.5 \%$ & $14.5 \%$ \\
\hline \multirow[t]{4}{*}{ Total } & $\mathrm{N}$ & 62 & 54 & 53 & 53 & 54 & 53 & 50 & 52 & 54 & 55 & 55 \\
\hline & Mean & 3.34 & 4.00 & 2.94 & 2.32 & 3.04 & 2.49 & 2.24 & 3.06 & 2.98 & 2.71 & 2.84 \\
\hline & Std. Deviation & 1.070 & .700 & \begin{tabular}{|l|l|}
1.027 \\
\end{tabular} & .872 & 1.027 & \begin{tabular}{|l|l|}
1.031 \\
\end{tabular} & 1.021 & 1.145 & 1.000 & 1.012 & \begin{tabular}{|l|l|}
1.067 \\
\end{tabular} \\
\hline & Variance & 1.146 & .491 & \begin{tabular}{|l|l|}
1.054 \\
\end{tabular} & .761 & 1.055 & 1.062 & 1.043 & 1.310 & 1.000 & 1.025 & 1.139 \\
\hline
\end{tabular}




\begin{tabular}{|l|l|l|l|l|l|l|l|l|l|l|l|l|}
\hline & Sum & 207 & 216 & 156 & 123 & 164 & 132 & 112 & 159 & 161 & 149 & 156 \\
\cline { 2 - 11 } & $\%$ of Total N & $100.0 \%$ & $100.0 \%$ & $100.0 \%$ & $100 \%$ & $100.0 \%$ & $100.0 \%$ & $100.0 \%$ & $100.0 \%$ & $100.0 \%$ & $100.0 \%$ & $100.0 \%$ \\
\hline Between groups (F-ratio) & 0.846 & $\begin{array}{l}3.933 * * \\
*\end{array}$ & 0.684 & 0.374 & 0.309 & 0.785 & 0.754 & $\begin{array}{l}4.003 * * \\
*\end{array}$ & 1.928 & 0.280 & 0.149 \\
\hline
\end{tabular}

Table 1. Continue

\begin{tabular}{|c|c|c|c|c|c|c|c|c|c|c|c|c|}
\hline & & 16 & 18. & 19. & 21. & 22. & 23. & 24. & 25. & 26. & 28. & 33. \\
\hline \multirow[t]{6}{*}{ Big Four } & $\mathrm{N}$ & 8 & 8 & 8 & 10 & 10 & 10 & 10 & 10 & 8 & 10 & 10 \\
\hline & Mean & 2.25 & 2.88 & 3.50 & 3.80 & 5.00 & 2.90 & 4.20 & 3.20 & 3.63 & 2.60 & 4.10 \\
\hline & Std. Deviation & 1.282 & 1.356 & 1.069 & 1.033 & 0.000 & 1.197 & 1.033 & 1.135 & 1.408 & 1.265 & .994 \\
\hline & Variance & 1.643 & 1.839 & 1.143 & 1.067 & 0.000 & 1.433 & 1.067 & 1.289 & 1.982 & 1.600 & .989 \\
\hline & Sum & 18 & 23 & 28 & 38 & 50 & 29 & 42 & 32 & 29 & 26 & 41 \\
\hline & $\%$ of Total $\mathrm{N}$ & $14.3 \%$ & $15.1 \%$ & $14.8 \%$ & $16.1 \%$ & $16.1 \%$ & $16.1 \%$ & $16.1 \%$ & $16.1 \%$ & $14.8 \%$ & $16.1 \%$ & $16.1 \%$ \\
\hline \multirow{6}{*}{$\begin{array}{l}\text { Other } \\
\text { Internatio } \\
\text { nal audit } \\
\text { network }\end{array}$} & $\mathrm{N}$ & 18 & 18 & 18 & 18 & 18 & 18 & 18 & 18 & 18 & 18 & 18 \\
\hline & Mean & 2.00 & 2.44 & 3.06 & 2.89 & 4.56 & 2.39 & 4.33 & 2.78 & 2.67 & 2.00 & 2.89 \\
\hline & Std. Deviation & .907 & .922 & .938 & 1.023 & .856 & .916 & 1.188 & 1.060 & .840 & 1.085 & 1.132 \\
\hline & Variance & .824 & .850 & .879 & 1.046 & .732 & .840 & 1.412 & 1.124 & .706 & 1.176 & 1.281 \\
\hline & Sum & 36 & 44 & 55 & 52 & 82 & 43 & 78 & 50 & 48 & 36 & 52 \\
\hline & $\%$ of Total $\mathrm{N}$ & $32.1 \%$ & $34.0 \%$ & $33.3 \%$ & $29.0 \%$ & $29.0 \%$ & $29.0 \%$ & $29.0 \%$ & $29.0 \%$ & $33.3 \%$ & $29.0 \%$ & $29.0 \%$ \\
\hline \multirow{6}{*}{$\begin{array}{l}\text { Local } \\
\text { audit } \\
\text { companie } \\
\text { s }\end{array}$} & $\mathrm{N}$ & 13 & 13 & 13 & 13 & 13 & 13 & 13 & 13 & 13 & 13 & 13 \\
\hline & Mean & 2.31 & 2.62 & 3.08 & 2.69 & 4.85 & 2.23 & 4.85 & 2.62 & 3.00 & 1.54 & 3.23 \\
\hline & Std. Deviation & .751 & 1.044 & .954 & 1.032 & .555 & 1.166 & .555 & 1.325 & 1.000 & .660 & 1.166 \\
\hline & Variance & .564 & 1.090 & .910 & 1.064 & .308 & 1.359 & .308 & 1.756 & 1.000 & .436 & 1.359 \\
\hline & Sum & 30 & 34 & 40 & 35 & 63 & 29 & 63 & 34 & 39 & 20 & 42 \\
\hline & $\%$ of Total $\mathrm{N}$ & $23.2 \%$ & $24.5 \%$ & $24.1 \%$ & $21.0 \%$ & $21.0 \%$ & $21.0 \%$ & $21.0 \%$ & $21.0 \%$ & $24.1 \%$ & $21.0 \%$ & $21.0 \%$ \\
\hline \multirow{6}{*}{$\begin{array}{l}\text { Certified } \\
\text { auditor - } \\
\text { sole } \\
\text { proprietor }\end{array}$} & $\mathrm{N}$ & 8 & 8 & 8 & 8 & 8 & 8 & 8 & 8 & 8 & 8 & 8 \\
\hline & Mean & 2.13 & 2.88 & 3.50 & 3.38 & 4.75 & 2.63 & 4.75 & 2.38 & 3.00 & 1.63 & 4.00 \\
\hline & Std. Deviation & 1.126 & .991 & .926 & 1.188 & .707 & 1.188 & .707 & 1.408 & .756 & .916 & 1.309 \\
\hline & Variance & 1.268 & .982 & .857 & 1.411 & .500 & 1.411 & .500 & 1.982 & .571 & .839 & 1.714 \\
\hline & Sum & 17 & 23 & 28 & 27 & 38 & 21 & 38 & 19 & 24 & 13 & 32 \\
\hline & $\%$ of Total $\mathrm{N}$ & $14.3 \%$ & $15.1 \%$ & $14.8 \%$ & $12.9 \%$ & $12.9 \%$ & $12.9 \%$ & $12.9 \%$ & $12.9 \%$ & $14.8 \%$ & $12.9 \%$ & $12.9 \%$ \\
\hline \multirow[t]{6}{*}{ Other } & $\mathrm{N}$ & 9 & 6 & 7 & 13 & 13 & 13 & 13 & 13 & 7 & 13 & 13 \\
\hline & Mean & 1.89 & 2.50 & 2.43 & 3.38 & 4.38 & 2.54 & 4.23 & 2.77 & 2.57 & 2.62 & 2.85 \\
\hline & Std. Deviation & .782 & .837 & .787 & 1.325 & 1.261 & 1.050 & 1.301 & 1.013 & 1.397 & 1.387 & 1.068 \\
\hline & Variance & .611 & .700 & .619 & 1.756 & 1.590 & 1.103 & 1.692 & 1.026 & 1.952 & 1.923 & 1.141 \\
\hline & Sum & 17 & 15 & 17 & 44 & 57 & 33 & 55 & 36 & 18 & 34 & 37 \\
\hline & $\%$ of Total $\mathrm{N}$ & $16.1 \%$ & $11.3 \%$ & $13.0 \%$ & $21.0 \%$ & $21.0 \%$ & $21.0 \%$ & $21.0 \%$ & $21.0 \%$ & $13.0 \%$ & $21.0 \%$ & $21.0 \%$ \\
\hline \multirow[t]{3}{*}{ Total } & $\mathrm{N}$ & 56 & 53 & 54 & 62 & 62 & 62 & 62 & 62 & 54 & 62 & 62 \\
\hline & Mean & 2.11 & 2.62 & 3.11 & 3.16 & 4.68 & 2.50 & 4.45 & 2.76 & 2.93 & 2.08 & 3.29 \\
\hline & Std. Deviation & .928 & 1.004 & .965 & 1.148 & .825 & 1.067 & 1.035 & 1.155 & 1.061 & 1.149 & 1.206 \\
\hline
\end{tabular}




\begin{tabular}{|c|c|c|c|c|c|c|c|c|c|c|c|c|}
\hline & Variance & .861 & 1.009 & .931 & 1.318 & .681 & 1.139 & 1.071 & 1.334 & 1.126 & 1.321 & 1.455 \\
\hline & Sum & 118 & 139 & 168 & 196 & 290 & 155 & 276 & 171 & 158 & 129 & 204 \\
\hline & $\%$ of Total $\mathrm{N}$ & $100.0 \%$ & $100.0 \%$ & $100.0 \%$ & $\begin{array}{l}100.0 \\
\%\end{array}$ & $100.0 \%$ & $100.0 \%$ & $100.0 \%$ & $100.0 \%$ & $100.0 \%$ & $100.0 \%$ & $100.0 \%$ \\
\hline Between g1 & oups (F-ratio) & 0.367 & 0.397 & 1.617 & 1.862 & 1.043 & 0.622 & 0.992 & 0.621 & 1.398 & $2.496 * *$ & $3.156 * *$ \\
\hline
\end{tabular}

Table 2. ANOVA analysis

\begin{tabular}{|c|c|c|c|c|c|c|}
\hline \multicolumn{7}{|l|}{ ANOVA } \\
\hline & & Sum of Squares & $\mathrm{df}$ & Mean Square & $\mathrm{F}$ & Sig. \\
\hline \multirow[t]{3}{*}{ 4. CHANGE } & Between Groups & 3.916 & 4 & .979 & .846 & .502 \\
\hline & Within Groups & 65.971 & 57 & 1.157 & & \\
\hline & Total & 69.887 & 61 & & & \\
\hline \multirow{3}{*}{$\begin{array}{l}5 . \\
\text { TRANSITION_WORKFROM } \\
\text { HOME }\end{array}$} & Between Groups & 6.319 & 4 & 1.580 & 3.933 & .008 \\
\hline & Within Groups & 19.681 & 49 & .402 & & \\
\hline & Total & 26.000 & 53 & & & \\
\hline \multirow[t]{3}{*}{ 7. UPDATING_AUDITPLAN } & Between Groups & 2.955 & 4 & .739 & .684 & .607 \\
\hline & Within Groups & 51.875 & 48 & 1.081 & & \\
\hline & Total & 54.830 & 52 & & & \\
\hline \multirow{3}{*}{$\begin{array}{l}8 . \\
\text { MATERIALITY_AUDITRISK }\end{array}$} & Between Groups & 1.195 & 4 & .299 & .374 & .826 \\
\hline & Within Groups & 38.353 & 48 & .799 & & \\
\hline & Total & 39.547 & 52 & & & \\
\hline \multirow[t]{3}{*}{ 9. AUDITEVIDENCE } & Between Groups & 1.374 & 4 & .344 & .309 & .871 \\
\hline & Within Groups & 54.552 & 49 & 1.113 & & \\
\hline & Total & 55.926 & 53 & & & \\
\hline \multirow{3}{*}{$\begin{array}{l}10 . \\
\text { ACCOUNTING_ESTIMATES }\end{array}$} & Between Groups & 3.393 & 4 & .848 & .785 & .540 \\
\hline & Within Groups & 51.853 & 48 & 1.080 & & \\
\hline & Total & 55.245 & 52 & & & \\
\hline \multirow[t]{3}{*}{ 11. CHANGE_PORTFOLIO } & Between Groups & 3.211 & 4 & .803 & .754 & .561 \\
\hline & Within Groups & 47.909 & 45 & 1.065 & & \\
\hline & Total & 51.120 & 49 & & & \\
\hline \multirow[t]{3}{*}{ 12. SCEPTICISM } & Between Groups & 16.983 & 4 & 4.246 & 4.003 & .007 \\
\hline & Within Groups & 49.844 & 47 & 1.061 & & \\
\hline & Total & 66.827 & 51 & & & \\
\hline \multirow[t]{3}{*}{ 13. INTERNALCONTROL } & Between Groups & 7.206 & 4 & 1.801 & 1.928 & .121 \\
\hline & Within Groups & 45.776 & 49 & .934 & & \\
\hline & Total & 52.981 & 53 & & & \\
\hline \multirow[t]{3}{*}{ 14. INCRFRAUD } & Between Groups & 1.212 & 4 & .303 & .280 & .890 \\
\hline & Within Groups & 54.134 & 50 & 1.083 & & \\
\hline & Total & 55.345 & 54 & & & \\
\hline
\end{tabular}




\begin{tabular}{|c|c|c|c|c|c|c|}
\hline \multirow[t]{3}{*}{ 15. REDFLAG } & Between Groups & .724 & 4 & .181 & .149 & .963 \\
\hline & Within Groups & 60.803 & 50 & 1.216 & & \\
\hline & Total & 61.527 & 54 & & & \\
\hline \multirow[t]{3}{*}{ 16. NOCLAR } & Between Groups & 1.324 & 4 & .331 & .367 & .831 \\
\hline & Within Groups & 46.033 & 51 & .903 & & \\
\hline & Total & 47.357 & 55 & & & \\
\hline \multirow[t]{3}{*}{ 18.AUDITREPORT_OPINION } & Between Groups & 1.681 & 4 & .420 & .397 & .809 \\
\hline & Within Groups & 50.771 & 48 & 1.058 & & \\
\hline & Total & 52.453 & 52 & & & \\
\hline \multirow[t]{3}{*}{ 19.AUDITREPORT_MATTER } & Between Groups & 5.752 & 4 & 1.438 & 1.617 & .185 \\
\hline & Within Groups & 43.582 & 49 & .889 & & \\
\hline & Total & 49.333 & 53 & & & \\
\hline \multirow{3}{*}{$\begin{array}{l}21 . \\
\text { STAKEHOLDER_EXPECTAT } \\
\text { IONS }\end{array}$} & Between Groups & 9.288 & 4 & 2.322 & 1.862 & .130 \\
\hline & Within Groups & 71.099 & 57 & 1.247 & & \\
\hline & Total & 80.387 & 61 & & & \\
\hline \multirow[t]{3}{*}{ 22. AUDITQUALITY } & Between Groups & 2.835 & 4 & .709 & 1.043 & .393 \\
\hline & Within Groups & 38.714 & 57 & .679 & & \\
\hline & Total & 41.548 & 61 & & & \\
\hline \multirow[t]{3}{*}{ 23. GAP_AUDITORS_USERS } & Between Groups & 2.909 & 4 & .727 & .622 & .648 \\
\hline & Within Groups & 66.591 & 57 & 1.168 & & \\
\hline & Total & 69.500 & 61 & & & \\
\hline \multirow[t]{3}{*}{ 24. QAAPROFESSION } & Between Groups & 4.255 & 4 & 1.064 & .992 & .419 \\
\hline & Within Groups & 61.100 & 57 & 1.072 & & \\
\hline & Total & 65.355 & 61 & & & \\
\hline \multirow[t]{3}{*}{ 25. WEDSERVICES } & Between Groups & 3.400 & 4 & .850 & .621 & .649 \\
\hline & Within Groups & 77.971 & 57 & 1.368 & & \\
\hline & Total & 81.371 & 61 & & & \\
\hline \multirow[t]{3}{*}{ 26.DECR_REVENUES } & Between Groups & 6.114 & 4 & 1.529 & 1.398 & .249 \\
\hline & Within Groups & 53.589 & 49 & 1.094 & & \\
\hline & Total & 59.704 & 53 & & & \\
\hline \multirow[t]{3}{*}{ 28. COMPR_ETHICS } & Between Groups & 12.014 & 4 & 3.004 & 2.496 & .053 \\
\hline & Within Groups & 68.583 & 57 & 1.203 & & \\
\hline & Total & 80.597 & 61 & & & \\
\hline \multirow{3}{*}{$\begin{array}{l}33 . \\
\text { POSTCOVID_SKILLSKNOW } \\
\text { L }\end{array}$} & Between Groups & 16.096 & 4 & 4.024 & 3.156 & .021 \\
\hline & Within Groups & 72.678 & 57 & 1.275 & & \\
\hline & Total & 88.774 & 61 & & & \\
\hline
\end{tabular}

The same results can be confirmed with performing nonparametric tests ok $\mathrm{K}$ independent samples through Kruskal Wallis Test (Table 3). 
Table 3. Nonparametric tests on $K$ independent samples

\begin{tabular}{|l|l|l|l|}
\hline & Chi-Square & df & Asymp. Sig. \\
\hline 4. CHANGE & 3.649 & 4 & .456 \\
\hline 5. TRANSITION_WORKFROMHOME & 12.882 & 4 & $.012^{*}$ \\
\hline 7. UPDATING_AUDITPLAN & 2.305 & 4 & .680 \\
\hline 8. MATERIALITY_AUDITRISK & .866 & 4 & .929 \\
\hline 9. AUDITEVIDENCE & 1.312 & 4 & .859 \\
\hline 10. ACCOUNTING_ESTIMATES & 2.072 & 4 & .722 \\
\hline 11. CHANGE_PORTFOLIO & 1.959 & 4 & .743 \\
\hline 12. SCEPTICISM & 12.669 & 4 & $.013^{*}$ \\
\hline 13. INTERNALCONTROL & 7.177 & 4 & .127 \\
\hline 14. INCRFRAUD & .842 & 4 & .933 \\
\hline 15. REDFLAG & .883 & 4 & .927 \\
\hline 16. NOCLAR & 1.553 & 4 & .817 \\
\hline 18.AUDITREPORT_OPINION & 1.515 & 4 & .824 \\
\hline 19. AUDITREPORT_MATTER & 5.978 & 4 & .201 \\
\hline 21. STAKEHOLDER_EXPECTATIONS & 7.630 & 4 & .106 \\
\hline 22. AUDITQUALITY & 3.853 & 4 & .426 \\
\hline 23. GAP_AUDITORS_USERS & 1.711 & .789 \\
\hline 24. QAAPROFESSION & 4.221 & .377 \\
\hline 25. WEDSERVICES & 3.316 & 4 & .506 \\
\hline 26.DECR_REVENUES & 5.027 & 4 & .285 \\
\hline 28. COMPR_ETHICS & 8.222 & $.084^{* * *}$ \\
\hline 33. POSTCOVID_SKILLSKNOWL & 11.192 & 4 & $.024^{* *}$ \\
\hline
\end{tabular}

Furthermore, Spearman's correlation (Appendix B) was carried out in order to examine the correlation between variables. A general conclusion is that for most of the variables there is no strong correlation, but moderate or low positive correlation. However, interesting is the result that the variable related with the audit report opinion and emphasizing paragraph have a strong correlation with the variable for expecting decreasing of the revenues during the pandemic period. Also, there is a strong correlation of the variable related with the auditors' perception of accounting estimations made by the management in the circumstances of uncertainty and variable related with favorable conditions for fraud triangle components and barriers for identification of red flags. These findings are a clear indication that auditors are aware that accounting estimations made in circumstances of uncertainty, risk, decreased resources, and difficult economic conditions as a whole can contribute to increasing fraud and difficulties to detect red flags.

\section{CONCLUSION}

The impacts of COVID-19 have forced external auditors around the world to seek answers to questions they may never have previously imagined. Auditors in the Republic of North Macedonia, as members of a small profession, have been operating for more than six months in the great shadow of the COVID-19 Pandemic. COVID-19 was the most rigorous test for the profession from which the public expects quality implementation of audit engagements in an environment we do not remember. As the world continues to seek answers to COVID-19 pandemic's global impact, through research conducted with certified auditors, members of the IORRM, we have come to good information about the vitality of the domestic profession. External auditors since the beginning of the pandemic have shown flexibility and vision on how to deal with the challenges posed by the 
pandemic. The results of the survey show that the auditors faced less impact of the pandemic on the audit of the financial statements for 2019 due to the fact that the pandemic followed the reporting period on December 31. For the financial statements for 2020, the auditors will focus on materiality, the risks of material misstatement, the risk factors of fraud related to the three elements of the fraud triangle, the issue of going concern, the manner of obtaining sufficient appropriate audit evidence in terms of remote work, assessment of accounting estimates, etc. According to the results of the research, it is realistic to expect the auditors to increase the level of professional skepticism, as a consequence of which the public will face a larger number of modified audit reports and more paragraphs to highlight the issue incorporated in them. Operating under the "new normal" according to the auditors will require different knowledge that should be part of the professional certification program. In general, the results of the research remind us that the challenges posed by the pandemic will be more easily overcome by joint and wise action of certified auditors, professional associations, supervisory bodies, and regulatory bodies in finding alternative ways not only to maintain routine activities but also to ways of acting in a radically changed environment under the influence of the COVID-19 Pandemic.

\section{REFERENCES}

ACCA, (2020). The impact of Covid-19 on Audit and Assurance - challenges and considerations, ACCA Thing Ahead.

Brachio, A., (2020). COVID-19: How to start looking forward with hope.

Dohrer, B. \& Mayes, C., (2020). 4 key COVID-19 audit risks for 2020 year ends.

GHRF Commission, (2016). The Neglected Dimension of Global Security: A Framework to

Counter Infectious Disease Crises, s.1.: Commission on a Global Health Risk Framework for the Future.

IFAC, (2020). Maintaining Trust \& Confidence During a Crisis - Lessons Learned from the Global COVID-19 Pandemic. [Online]

Available at: https://www.ifac.org/what-we-do/speak-out-global-voice/points-view/maintainingtrust-confidence-during-crisis

IFAC, (2020). Public Interest Responsibilities Must Remain Paramount in an Age of COVID-19, s.1.: s.n.

IOSCO, (2020). Statement on Importance of Disclosure about COVID-19. 


\section{APPENDIX A}

Table A-1. Descriptive statistics of the responses

\begin{tabular}{|c|c|c|c|c|c|c|c|c|}
\hline No. & Question & $\mathrm{N}$ & Mean & $\begin{array}{l}\text { Std. } \\
\text { Deviation }\end{array}$ & Variance & Min & Max & Sum \\
\hline 4 & $\begin{array}{l}\text { How much has the COVID-19 Pandemic changed the } \\
\text { approach to your professional engagement? }\end{array}$ & 62 & 3.34 & 1.070 & 1.146 & 1 & 5 & 207 \\
\hline 5 & $\begin{array}{l}\text { In the new circumstances initiated by the COVID-19 } \\
\text { Pandemic, was your audit firm prepared to implement the } \\
\text { IFAC recommendations for the transition from the classic } \\
\text { office to the remote environment in order to protect the health } \\
\text { and lives of employees? }\end{array}$ & 54 & 4.00 & .700 & .491 & 3 & 5 & 216 \\
\hline 7 & $\begin{array}{l}\text { Given your client portfolio, did the uncertainties imposed by } \\
\text { the COVID-19 Pandemic reflect your judgment in updating } \\
\text { the audit plan of certain audited entities? }\end{array}$ & 53 & 2.94 & 1.027 & 1.054 & 1 & 5 & 156 \\
\hline 8 & $\begin{array}{l}\text { The uncertainties imposed by the COVID-19 Pandemic affect } \\
\text { your professional judgment in the segment of reviewing the } \\
\text { preliminary judgment about materiality and audit risk of } \\
\text { certain audited entities? }\end{array}$ & 53 & 2.32 & .872 & .761 & 1 & 4 & 123 \\
\hline 9 & $\begin{array}{l}\text { Working in conditions of quarantine measures, self-isolation, } \\
\text { and remote work influenced the application of audit } \\
\text { procedures for collecting and documenting sufficiently } \\
\text { appropriate audit evidence in existing and especially in new } \\
\text { audit entities? }\end{array}$ & 54 & 3.04 & 1.027 & 1.055 & 1 & 5 & 164 \\
\hline 10 & $\begin{array}{l}\text { Working remotely affects your perception of the accounting } \\
\text { estimates that were made in a time of uncertainty by the } \\
\text { management of the audited entities or by using the work of } \\
\text { experts in areas that require special knowledge? }\end{array}$ & 53 & 2.49 & 1.031 & 1.062 & 1 & 5 & 132 \\
\hline 11 & $\begin{array}{l}\text { As a consequence of the Pandemic with COVID-19 do you } \\
\text { expect that the portfolio of your clients will change due to } \\
\text { inability to work according to the going concern principle } \\
\text { (clients from certain industries most sensitive to the measures } \\
\text { taken to deal with the Pandemic such as restaurants, hotels, } \\
\text { etc.)? }\end{array}$ & 50 & 2.24 & 1.021 & 1.043 & 1 & 5 & 112 \\
\hline 12 & $\begin{array}{l}\text { The health crisis initiated by the COVID-19 Pandemic and its } \\
\text { overflow in the economy with complete closure of enterprises } \\
\text { and their gradual opening, dismissal of employees, etc. } \\
\text { influenced you to increase the level of professional skepticism } \\
\text { during the audit engagements, due to the increased risk of } \\
\text { material misstatement in the financial statements as a result } \\
\text { of fraud or error? }\end{array}$ & 52 & 3.06 & 1.145 & 1.310 & 1 & 5 & 159 \\
\hline 13 & $\begin{array}{l}\text { Closing of clients, rotation of employees, application of } \\
\text { quarantine measures supplemented by remote work, enabled } \\
\text { possible modifications in the design and functioning of the } \\
\text { elements of internal control in the auditing entities? }\end{array}$ & 54 & 2.98 & 1.000 & 1.000 & 1 & 5 & 161 \\
\hline 14 & $\begin{array}{l}\text { In times of crisis, such as the COVID-19 Pandemic, fraud risk } \\
\text { factors become more numerous as companies and } \\
\text { management face greater financial pressures, opportunities } \\
\text { for fraud increase due to weaknesses in the functioning of } \\
\text { internal controls, and individuals are more easily to } \\
\text { rationalize their fraudulent activities. COVID-19 created } \\
\text { favorable conditions for all three elements of the fraud } \\
\text { triangle - even more than that - to be present in the operations } \\
\text { of business entities? }\end{array}$ & 55 & 2.71 & 1.012 & 1.025 & 1 & 5 & 149 \\
\hline 15 & $\begin{array}{l}\text { In your opinion, working remotely even in case of an } \\
\text { increased level of professional skepticism imposed certain } \\
\text { barriers in identifying the symptoms of fraud (so-called "red } \\
\text { flags") due to the impossibility of conducting an interview to } \\
\text { establish closer contact with employees and to does body } \\
\text { language follow? }\end{array}$ & 55 & 2.84 & 1.067 & 1.139 & 1 & 5 & 156 \\
\hline
\end{tabular}




\begin{tabular}{|c|c|c|c|c|c|c|c|c|}
\hline 16 & $\begin{array}{l}\text { In your opinion, will the new environment imposed by } \\
\text { COVID-19 tempt the management of audited entities to } \\
\text { disregard laws and regulations more often (NOCLAR - Non- } \\
\text { Compliance with Law and Regulation)? }\end{array}$ & 56 & 2.11 & .928 & .861 & 1 & 5 & 118 \\
\hline 18 & $\begin{array}{l}\text { In your opinion, the COVID-19 Pandemic will affect the form } \\
\text { and content of the audit report in terms of increased number } \\
\text { of modifications in the expressed audit opinion } \\
\text { (Unqualified opinion, Qualified opinion, Disclaimer } \\
\text { of opinion, or Adverse opinion) for the audited financial } \\
\text { statements of the entities for the year ending } 31.12 .2020 \text { ? }\end{array}$ & 53 & 2.62 & 1.004 & 1.009 & 1 & 5 & 139 \\
\hline 19 & $\begin{array}{l}\text { In your opinion, will the work of the auditing entities in } \\
\text { conditions of a COVID- } 19 \text { Pandemic affect the more frequent } \\
\text { use of paragraphs for emphasis of matters in the audit reports } \\
\text { for presented financial statements for the year ended } \\
31.12 .2020 \text { ? }\end{array}$ & 54 & 3.11 & .965 & .931 & 2 & 5 & 168 \\
\hline 21 & $\begin{array}{l}\text { The COVID-19 Pandemic more than ever raised the } \\
\text { expectations of investors and other stakeholders for } \\
\text { presenting quality financial statements and conducting } \\
\text { quality audits? }\end{array}$ & 62 & 3.16 & 1.148 & 1.318 & 1 & 5 & 196 \\
\hline 22 & $\begin{array}{l}\text { In conditions of remote work, limited communication with } \\
\text { the management and employees of the client, closure of the } \\
\text { offices of the auditees, quarantine measures introduced, } \\
\text { supply chain interruptions, and complete paralysis of the } \\
\text { work of the auditees, the auditors must not sacrifice the } \\
\text { quality of the audit? }\end{array}$ & 62 & 4.68 & .825 & .681 & 1 & 5 & 290 \\
\hline 23 & $\begin{array}{l}\text { The COVID-19 Pandemic will enlarge the expectation gap } \\
\text { between certified auditors, on the one hand, and users of } \\
\text { audited financial statements, on the other? }\end{array}$ & 62 & 2.50 & 1.067 & 1.139 & 1 & 5 & 155 \\
\hline 24 & $\begin{array}{l}\text { The risks posed by the COVID-19 Pandemic in terms of true } \\
\text { and fair presentation of the financial statements, accent the } \\
\text { importance of having a quality accounting and auditing } \\
\text { profession? }\end{array}$ & 62 & 4.45 & 1.035 & 1.071 & 1 & 5 & 276 \\
\hline 25 & $\begin{array}{l}\text { The COVID-19 Pandemic will expand the range of services } \\
\text { that existing and potential customers will require? }\end{array}$ & 62 & 2.76 & 1.155 & 1.334 & 1 & 5 & 171 \\
\hline 26 & $\begin{array}{l}\text { Work in an environment that is constantly changing under the } \\
\text { influence of the prolonged first wave of the Pandemic in the } \\
\text { summer months and the second wave announced by the } \\
\text { World Health Organization by the end of the year, will } \\
\text { adversely affect the results of auditors which may reduce your } \\
\text { revenue based on audit services? }\end{array}$ & 54 & 2.93 & 1.061 & 1.126 & 1 & 5 & 158 \\
\hline 28 & $\begin{array}{l}\text { Do you think that the uncertain environment in which } \\
\text { business entities operate in the face of the COVID-19 } \\
\text { Pandemic will tempt auditors to compromise the basic } \\
\text { principles of the International Code of Ethics for Professional } \\
\text { Accountants? }\end{array}$ & 62 & 2.08 & 1.149 & 1.321 & 1 & 5 & 129 \\
\hline 33 & $\begin{array}{l}\text { In the post-COVID period more than ever before, auditing } \\
\text { firms will need different skills and knowledge to be } \\
\text { incorporated into professional programs? }\end{array}$ & 62 & 3.29 & 1.206 & 1.455 & 1 & 5 & 204 \\
\hline
\end{tabular}


Table B-1. Spearman correlation

\begin{tabular}{|c|c|c|c|c|c|c|c|c|c|c|c|c|c|}
\hline \multicolumn{14}{|c|}{ Correlations } \\
\hline & & 4 & 5 & 7 & 8 & 9 & 10 & 11 & 12 & 13 & 14 & 15 & 16 \\
\hline \multirow[t]{3}{*}{4} & $\begin{array}{l}\text { Correlation } \\
\text { Coefficient }\end{array}$ & 1.000 & $.292^{*}$ & $.297^{*}$ & .170 & $.316^{*}$ & .063 & .128 & .148 & $.439^{* *}$ & -.055 & $.340^{*}$ & .093 \\
\hline & $\begin{array}{l}\text { Sig. (2- } \\
\text { tailed) }\end{array}$ & & .032 & .031 & .224 & .020 & .654 & .378 & .294 & .001 & .692 & .011 & .495 \\
\hline & $\mathrm{N}$ & 62 & 54 & 53 & 53 & 54 & 53 & 50 & 52 & 54 & 55 & 55 & 56 \\
\hline \multirow[t]{3}{*}{5} & $\begin{array}{l}\text { Correlation } \\
\text { Coefficient } \\
\end{array}$ & $.292^{*}$ & 1.000 & -.142 & -.024 & .080 & .115 & $\begin{array}{l}-.097 \\
\end{array}$ & .236 & $.307^{*}$ & -.093 & .114 & .113 \\
\hline & $\begin{array}{l}\text { Sig. (2- } \\
\text { tailed) }\end{array}$ & .032 & & .310 & .866 & .566 & .414 & .502 & .092 & .025 & .508 & .412 & .418 \\
\hline & $\mathrm{N}$ & 54 & 54 & 53 & 53 & 54 & 53 & 50 & 52 & 53 & 53 & 54 & 54 \\
\hline \multirow[t]{3}{*}{7} & $\begin{array}{l}\text { Correlation } \\
\text { Coefficient }\end{array}$ & $.297^{*}$ & -.142 & 1.000 & $.426^{* *}$ & $.303^{*}$ & .092 & .224 & .049 & $.279^{*}$ & .060 & $.275^{*}$ & .205 \\
\hline & $\begin{array}{l}\text { Sig. (2- } \\
\text { tailed) }\end{array}$ & .031 & .310 & & .002 & .027 & .517 & .122 & .731 & .045 & .674 & .046 & .141 \\
\hline & $\mathrm{N}$ & 53 & 53 & 53 & 52 & 53 & 52 & 49 & 51 & 52 & 52 & 53 & 53 \\
\hline \multirow[t]{3}{*}{8} & $\begin{array}{l}\text { Correlation } \\
\text { Coefficient }\end{array}$ & .170 & -.024 & $.426^{* *}$ & 1.000 & $.300^{*}$ & .230 & $.305^{*}$ & .211 & $.299^{*}$ & .200 & $.442^{* *}$ & .165 \\
\hline & $\begin{array}{l}\text { Sig. (2- } \\
\text { tailed) } \\
\end{array}$ & .224 & .866 & .002 & & .029 & .100 & .031 & .138 & .031 & .156 & .001 & .239 \\
\hline & $\mathrm{N}$ & 53 & 53 & 52 & 53 & 53 & 52 & 50 & 51 & 52 & 52 & 53 & 53 \\
\hline \multirow[t]{3}{*}{9} & $\begin{array}{l}\text { Correlation } \\
\text { Coefficient }\end{array}$ & $.316^{*}$ & .080 & $.303^{*}$ & $.300^{*}$ & 1.000 & $.481^{* *}$ & .139 & .144 & $.274^{*}$ & $.414^{* *}$ & $.474^{* *}$ & $.308^{*}$ \\
\hline & $\begin{array}{l}\text { Sig. (2- } \\
\text { tailed) }\end{array}$ & .020 & .566 & .027 & .029 & & .000 & .336 & .310 & .047 & .002 & .000 & .024 \\
\hline & $\mathrm{N}$ & 54 & 54 & 53 & 53 & 54 & 53 & 50 & 52 & 53 & 53 & 54 & 54 \\
\hline \multirow[t]{3}{*}{10} & $\begin{array}{l}\text { Correlation } \\
\text { Coefficient }\end{array}$ & .063 & .115 & .092 & .230 & $.481^{* *}$ & 1.000 & $.400^{* *}$ & $.283^{*}$ & $.378^{* *}$ & $.445^{* *}$ & $.483^{* *}$ & .254 \\
\hline & $\begin{array}{l}\text { Sig. (2- } \\
\text { tailed) }\end{array}$ & .654 & .414 & .517 & .100 & .000 & & .004 & .042 & .005 & .001 & .000 & .066 \\
\hline & $\mathrm{N}$ & 53 & 53 & 52 & 52 & 53 & 53 & 50 & 52 & 53 & 53 & 53 & 53 \\
\hline \multirow[t]{3}{*}{11} & $\begin{array}{l}\text { Correlation } \\
\text { Coefficient }\end{array}$ & .128 & -.097 & .224 & $.305^{*}$ & .139 & $.400^{* *}$ & 1.000 & $.405^{* *}$ & .146 & $.413^{* *}$ & .198 & -.026 \\
\hline & $\begin{array}{l}\text { Sig. (2- } \\
\text { tailed) }\end{array}$ & .378 & .502 & .122 & .031 & .336 & .004 & & .003 & .310 & .003 & .169 & .858 \\
\hline & $\mathrm{N}$ & 50 & 50 & 49 & 50 & 50 & 50 & 50 & 50 & 50 & 50 & 50 & 50 \\
\hline \multirow[t]{3}{*}{12} & $\begin{array}{l}\text { Correlation } \\
\text { Coefficient }\end{array}$ & .148 & .236 & .049 & .211 & .144 & $.283^{*}$ & $.405^{* *}$ & 1.000 & $.361^{* *}$ & .254 & -.057 & .001 \\
\hline & $\begin{array}{l}\text { Sig. (2- } \\
\text { tailed) }\end{array}$ & .294 & .092 & .731 & .138 & .310 & .042 & .003 & & .009 & .070 & .686 & .996 \\
\hline & $\mathrm{N}$ & 52 & 52 & 51 & 51 & 52 & 52 & 50 & 52 & 52 & 52 & 52 & 52 \\
\hline \multirow[t]{3}{*}{13} & $\begin{array}{l}\text { Correlation } \\
\text { Coefficient }\end{array}$ & $.439^{* *}$ & $.307^{*}$ & $.279^{*}$ & $.299^{*}$ & $.274^{*}$ & $.378^{* *}$ & .146 & $.361^{* *}$ & 1.000 & $.282^{*}$ & $.437^{* *}$ & $.286^{*}$ \\
\hline & $\begin{array}{l}\text { Sig. (2- } \\
\text { tailed) }\end{array}$ & .001 & .025 & .045 & .031 & .047 & .005 & .310 & .009 & & .039 & .001 & .036 \\
\hline & $\mathrm{N}$ & 54 & 53 & 52 & 52 & 53 & 53 & 50 & 52 & 54 & 54 & 54 & 54 \\
\hline 14 & $\begin{array}{l}\text { Correlation } \\
\text { Coefficient }\end{array}$ & -.055 & -.093 & .060 & .200 & $.414^{* *}$ & $.445^{* *}$ & $.413^{* *}$ & .254 & $.282^{*}$ & 1.000 & $.335^{*}$ & .160 \\
\hline
\end{tabular}




\begin{tabular}{|c|c|c|c|c|c|c|c|c|c|c|c|c|c|}
\hline & $\begin{array}{l}\text { Sig. (2- } \\
\text { tailed) }\end{array}$ & .692 & .508 & .674 & .156 & .002 & .001 & .003 & .070 & .039 & & .013 & .244 \\
\hline & $\mathrm{N}$ & 55 & 53 & 52 & 52 & 53 & 53 & 50 & 52 & 54 & 55 & 54 & 55 \\
\hline \multirow[t]{3}{*}{15} & $\begin{array}{l}\text { Correlation } \\
\text { Coefficient }\end{array}$ & $.340^{*}$ & .114 & $.275^{*}$ & $.442^{* *}$ & $.474^{* *}$ & $.483^{* *}$ & .198 & -.057 & $.437^{* *}$ & $.335^{*}$ & 1.000 & $.423^{* *}$ \\
\hline & $\begin{array}{l}\text { Sig. (2- } \\
\text { tailed) }\end{array}$ & .011 & .412 & .046 & .001 & .000 & .000 & .169 & .686 & .001 & .013 & & .001 \\
\hline & $\mathrm{N}$ & 55 & 54 & 53 & 53 & 54 & 53 & 50 & 52 & 54 & 54 & 55 & 55 \\
\hline \multirow[t]{3}{*}{16} & $\begin{array}{l}\text { Correlation } \\
\text { Coefficient }\end{array}$ & .093 & .113 & .205 & .165 & $.308^{*}$ & .254 & -.026 & .001 & $.286^{*}$ & .160 & $.423^{* *}$ & 1.000 \\
\hline & $\begin{array}{l}\text { Sig. (2- } \\
\text { tailed) }\end{array}$ & .495 & .418 & .141 & .239 & .024 & .066 & .858 & .996 & .036 & .244 & .001 & \\
\hline & $\mathrm{N}$ & 56 & 54 & 53 & 53 & 54 & 53 & 50 & 52 & 54 & 55 & 55 & 56 \\
\hline \multirow[t]{3}{*}{18} & $\begin{array}{l}\text { Correlation } \\
\text { Coefficient }\end{array}$ & .032 & .132 & .033 & .159 & $.358^{* *}$ & $.348^{*}$ & .212 & .177 & .259 & $.362^{* *}$ & $.363^{* *}$ & $.326^{*}$ \\
\hline & $\begin{array}{l}\text { Sig. (2- } \\
\text { tailed) }\end{array}$ & .822 & .354 & .818 & .269 & .010 & .012 & .140 & .214 & .064 & .008 & .008 & .017 \\
\hline & $\mathrm{N}$ & 53 & 51 & 50 & 50 & 51 & 51 & 50 & 51 & 52 & 53 & 52 & 53 \\
\hline \multirow[t]{3}{*}{19} & $\begin{array}{l}\text { Correlation } \\
\text { Coefficient }\end{array}$ & .007 & .044 & .174 & .198 & $.417^{* *}$ & $.324^{*}$ & .186 & $.309^{*}$ & .130 & .253 & .189 & .267 \\
\hline & $\begin{array}{l}\text { Sig. (2- } \\
\text { tailed) }\end{array}$ & .957 & .755 & .221 & .164 & .002 & .019 & .196 & .026 & .354 & .065 & .175 & .051 \\
\hline & $\mathrm{N}$ & 54 & 52 & 51 & 51 & 52 & 52 & 50 & 52 & 53 & 54 & 53 & 54 \\
\hline \multirow[t]{3}{*}{21} & $\begin{array}{l}\text { Correlation } \\
\text { Coefficient }\end{array}$ & $.268^{*}$ & .185 & .165 & $.371^{* *}$ & .058 & .164 & $.366^{* *}$ & $.400^{* *}$ & $.413^{* *}$ & .204 & $.300^{*}$ & .098 \\
\hline & $\begin{array}{l}\text { Sig. (2- } \\
\text { tailed) }\end{array}$ & .035 & .180 & .238 & .006 & .678 & .239 & .009 & .003 & .002 & .134 & .026 & .472 \\
\hline & $\mathrm{N}$ & 62 & 54 & 53 & 53 & 54 & 53 & 50 & 52 & 54 & 55 & 55 & 56 \\
\hline \multirow[t]{3}{*}{22} & $\begin{array}{l}\text { Correlation } \\
\text { Coefficient }\end{array}$ & .224 & .238 & .029 & .075 & .063 & .052 & .177 & .176 & $.280^{*}$ & -.098 & .032 & .079 \\
\hline & $\begin{array}{l}\text { Sig. (2- } \\
\text { tailed) }\end{array}$ & .080 & .083 & .839 & .596 & .651 & .713 & .218 & .211 & .040 & .475 & .816 & .561 \\
\hline & $\mathrm{N}$ & 62 & 54 & 53 & 53 & 54 & 53 & 50 & 52 & 54 & 55 & 55 & 56 \\
\hline \multirow[t]{3}{*}{23} & $\begin{array}{l}\text { Correlation } \\
\text { Coefficient }\end{array}$ & .185 & .117 & $.280^{*}$ & $.320^{*}$ & $.344^{*}$ & $.548^{* *}$ & $.359^{*}$ & $.358^{* *}$ & .257 & .259 & $.300^{*}$ & $.391^{* *}$ \\
\hline & $\begin{array}{l}\text { Sig. (2- } \\
\text { tailed) }\end{array}$ & .150 & .401 & .042 & .020 & .011 & .000 & .010 & .009 & .060 & .057 & .026 & .003 \\
\hline & $\mathrm{N}$ & 62 & 54 & 53 & 53 & 54 & 53 & 50 & 52 & 54 & 55 & 55 & 56 \\
\hline \multirow[t]{3}{*}{24} & $\begin{array}{l}\text { Correlation } \\
\text { Coefficient }\end{array}$ & -.183 & .137 & .091 & .143 & -.028 & .179 & .012 & .025 & $.321^{*}$ & .134 & .132 & .211 \\
\hline & $\begin{array}{l}\text { Sig. (2- } \\
\text { tailed) }\end{array}$ & .154 & .322 & .518 & .306 & .843 & .199 & .933 & .858 & .018 & .328 & .337 & .119 \\
\hline & $\mathrm{N}$ & 62 & 54 & 53 & 53 & 54 & 53 & 50 & 52 & 54 & 55 & 55 & 56 \\
\hline \multirow[t]{3}{*}{25} & $\begin{array}{l}\text { Correlation } \\
\text { Coefficient }\end{array}$ & .146 & .206 & .265 & .221 & .089 & .095 & .017 & .002 & .118 & .047 & .059 & .195 \\
\hline & $\begin{array}{l}\text { Sig. (2- } \\
\text { tailed) }\end{array}$ & .258 & .136 & .055 & .112 & .522 & .497 & .907 & .988 & .396 & .734 & .669 & .149 \\
\hline & $\mathrm{N}$ & 62 & 54 & 53 & 53 & 54 & 53 & 50 & 52 & 54 & 55 & 55 & 56 \\
\hline \multirow[t]{3}{*}{26} & $\begin{array}{l}\text { Correlation } \\
\text { Coefficient } \\
\end{array}$ & -.001 & .189 & .015 & $.361^{* *}$ & .257 & $.394^{* *}$ & .147 & $.338^{*}$ & $.367^{* *}$ & $.332^{*}$ & .246 & .153 \\
\hline & $\begin{array}{l}\text { Sig. (2- } \\
\text { tailed) }\end{array}$ & .993 & .179 & .915 & .009 & .066 & .004 & .309 & .015 & .007 & .016 & .076 & .275 \\
\hline & $\mathrm{N}$ & 54 & 52 & 51 & 51 & 52 & 51 & 50 & 51 & 52 & 52 & 53 & 53 \\
\hline 28 & $\begin{array}{l}\text { Correlation } \\
\text { Coefficient }\end{array}$ & .219 & .206 & .251 & .252 & $.438^{* *}$ & .162 & -.089 & .164 & $.561^{* *}$ & .095 & $.328^{*}$ & .241 \\
\hline
\end{tabular}




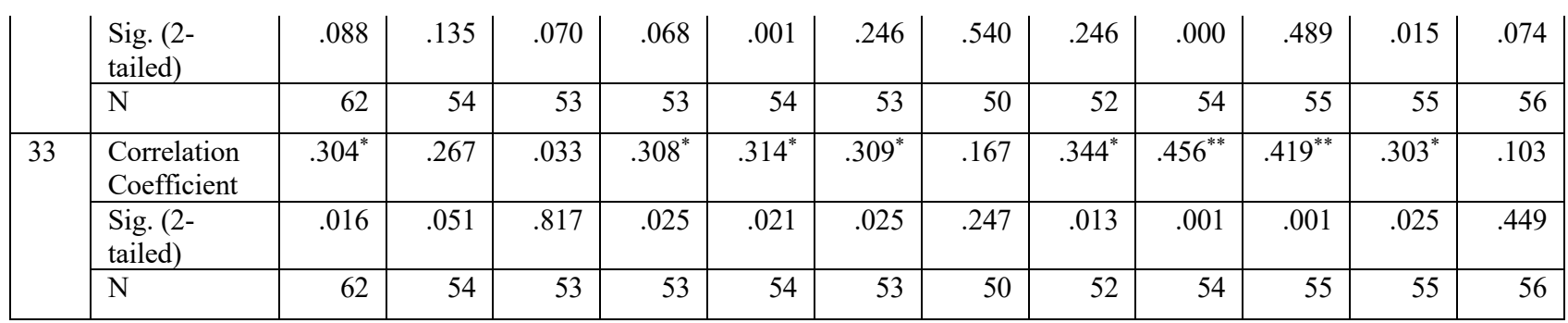

*. Correlation is significant at the 0.05 level (2-tailed).

**. Correlation is significant at the 0.01 level (2-tailed).

Table B-2. Continue

\begin{tabular}{|c|c|c|c|c|c|c|c|c|c|c|c|}
\hline \multicolumn{12}{|c|}{ Correlations } \\
\hline & & 18 & 19 & 21 & 22 & 23 & 24 & 25 & 26 & 28 & 33 \\
\hline \multirow[t]{3}{*}{4} & $\begin{array}{l}\text { Correlation } \\
\text { Coefficient }\end{array}$ & .032 & .007 & $.268^{*}$ & .224 & .185 & -.183 & .146 & -.001 & .219 & $.304^{*}$ \\
\hline & Sig. (2-tailed) & .822 & .957 & .035 & .080 & .150 & .154 & .258 & .993 & .088 & .016 \\
\hline & $\mathrm{N}$ & 53 & 54 & 62 & 62 & 62 & 62 & 62 & 54 & 62 & 62 \\
\hline \multirow[t]{3}{*}{5} & $\begin{array}{l}\text { Correlation } \\
\text { Coefficient }\end{array}$ & .132 & .044 & .185 & .238 & .117 & .137 & .206 & .189 & .206 & .267 \\
\hline & Sig. (2-tailed) & .354 & .755 & .180 & .083 & .401 & .322 & .136 & .179 & .135 & .051 \\
\hline & $\mathrm{N}$ & 51 & 52 & 54 & 54 & 54 & 54 & 54 & 52 & 54 & 54 \\
\hline \multirow[t]{3}{*}{7} & $\begin{array}{l}\text { Correlation } \\
\text { Coefficient }\end{array}$ & .033 & .174 & .165 & .029 & $.280^{*}$ & .091 & .265 & .015 & .251 & .033 \\
\hline & Sig. (2-tailed) & .818 & .221 & .238 & .839 & .042 & .518 & .055 & .915 & .070 & .817 \\
\hline & $\mathrm{N}$ & 50 & 51 & 53 & 53 & 53 & 53 & 53 & 51 & 53 & 53 \\
\hline \multirow[t]{3}{*}{8} & $\begin{array}{l}\text { Correlation } \\
\text { Coefficient }\end{array}$ & .159 & .198 & $.371^{* *}$ & .075 & $.320^{*}$ & .143 & .221 & $.361^{* *}$ & .252 & $.308^{*}$ \\
\hline & Sig. (2-tailed) & .269 & .164 & .006 & .596 & .020 & .306 & .112 & .009 & .068 & .025 \\
\hline & $\mathrm{N}$ & 50 & 51 & 53 & 53 & 53 & 53 & 53 & 51 & 53 & 53 \\
\hline \multirow[t]{3}{*}{9} & $\begin{array}{l}\text { Correlation } \\
\text { Coefficient }\end{array}$ & $.358^{* *}$ & $.417^{* *}$ & .058 & .063 & $.344^{*}$ & -.028 & .089 & .257 & $.438^{* *}$ & $.314^{*}$ \\
\hline & Sig. (2-tailed) & .010 & .002 & .678 & .651 & .011 & .843 & .522 & .066 & .001 & .021 \\
\hline & $\mathrm{N}$ & 51 & 52 & 54 & 54 & 54 & 54 & 54 & 52 & 54 & 54 \\
\hline \multirow[t]{3}{*}{10} & $\begin{array}{l}\text { Correlation } \\
\text { Coefficient }\end{array}$ & $.348^{*}$ & $.324^{*}$ & .164 & .052 & $.548^{* *}$ & .179 & .095 & $.394^{* *}$ & .162 & $.309^{*}$ \\
\hline & Sig. (2-tailed) & .012 & .019 & .239 & .713 & .000 & .199 & .497 & .004 & .246 & .025 \\
\hline & $\mathrm{N}$ & 51 & 52 & 53 & 53 & 53 & 53 & 53 & 51 & 53 & 53 \\
\hline \multirow[t]{3}{*}{11} & $\begin{array}{l}\text { Correlation } \\
\text { Coefficient }\end{array}$ & .212 & .186 & $.366^{* *}$ & .177 & $.359^{*}$ & .012 & .017 & .147 & -.089 & .167 \\
\hline & Sig. (2-tailed) & .140 & .196 & .009 & .218 & .010 & .933 & .907 & .309 & .540 & .247 \\
\hline & $\mathrm{N}$ & 50 & 50 & 50 & 50 & 50 & 50 & 50 & 50 & 50 & 50 \\
\hline \multirow[t]{2}{*}{12} & $\begin{array}{l}\text { Correlation } \\
\text { Coefficient }\end{array}$ & .177 & $.309^{*}$ & $.400^{* *}$ & .176 & $.358^{* *}$ & .025 & .002 & $.338^{*}$ & .164 & $.344^{*}$ \\
\hline & Sig. (2-tailed) & .214 & .026 & .003 & .211 & .009 & .858 & .988 & .015 & .246 & .013 \\
\hline
\end{tabular}




\begin{tabular}{|c|c|c|c|c|c|c|c|c|c|c|c|}
\hline & $\mathrm{N}$ & 51 & 52 & 52 & 52 & 52 & 52 & 52 & 51 & 52 & 52 \\
\hline \multirow[t]{3}{*}{13} & $\begin{array}{l}\text { Correlation } \\
\text { Coefficient }\end{array}$ & .259 & .130 & $.413^{* *}$ & $.280^{*}$ & .257 & $.321^{*}$ & .118 & $.367^{* *}$ & $.561^{* *}$ & $.456^{* *}$ \\
\hline & Sig. (2-tailed) & .064 & .354 & .002 & .040 & .060 & .018 & .396 & .007 & .000 & .001 \\
\hline & $\mathrm{N}$ & 52 & 53 & 54 & 54 & 54 & 54 & 54 & 52 & 54 & 54 \\
\hline \multirow[t]{3}{*}{14} & $\begin{array}{l}\text { Correlation } \\
\text { Coefficient }\end{array}$ & $.362^{* *}$ & .253 & .204 & -.098 & .259 & .134 & .047 & $.332^{*}$ & .095 & $.419^{* *}$ \\
\hline & Sig. (2-tailed) & .008 & .065 & .134 & .475 & .057 & .328 & .734 & .016 & .489 & .001 \\
\hline & $\mathrm{N}$ & 53 & 54 & 55 & 55 & 55 & 55 & 55 & 52 & 55 & 55 \\
\hline \multirow[t]{3}{*}{15} & $\begin{array}{l}\text { Correlation } \\
\text { Coefficient }\end{array}$ & $.363^{* *}$ & .189 & $.300^{*}$ & .032 & $.300^{*}$ & .132 & .059 & .246 & $.328^{*}$ & $.303^{*}$ \\
\hline & Sig. (2-tailed) & .008 & .175 & .026 & .816 & .026 & .337 & .669 & .076 & .015 & .025 \\
\hline & $\mathrm{N}$ & 52 & 53 & 55 & 55 & 55 & 55 & 55 & 53 & 55 & 55 \\
\hline \multirow[t]{3}{*}{16} & $\begin{array}{l}\text { Correlation } \\
\text { Coefficient }\end{array}$ & $.326^{*}$ & .267 & .098 & .079 & $.391^{* *}$ & .211 & .195 & .153 & .241 & .103 \\
\hline & Sig. (2-tailed) & .017 & .051 & .472 & .561 & .003 & .119 & .149 & .275 & .074 & .449 \\
\hline & $\mathrm{N}$ & 53 & 54 & 56 & 56 & 56 & 56 & 56 & 53 & 56 & 56 \\
\hline \multirow[t]{3}{*}{18} & $\begin{array}{l}\text { Correlation } \\
\text { Coefficient }\end{array}$ & 1.000 & $.724^{* *}$ & $.319^{*}$ & -.186 & $.433^{* *}$ & $.279^{*}$ & .224 & $.523^{* *}$ & .219 & $.383^{* *}$ \\
\hline & Sig. (2-tailed) & & .000 & .020 & .181 & .001 & .043 & .107 & .000 & .116 & .005 \\
\hline & $\mathrm{N}$ & 53 & 53 & 53 & 53 & 53 & 53 & 53 & 52 & 53 & 53 \\
\hline \multirow[t]{3}{*}{19} & $\begin{array}{l}\text { Correlation } \\
\text { Coefficient }\end{array}$ & $.724^{* *}$ & 1.000 & $.307^{*}$ & -.166 & $.328^{*}$ & .079 & $.306^{*}$ & $.472^{* *}$ & .265 & $.485^{* *}$ \\
\hline & Sig. (2-tailed) & .000 & & .024 & .230 & .015 & .572 & .024 & .000 & .053 & .000 \\
\hline & $\mathrm{N}$ & 53 & 54 & 54 & 54 & 54 & 54 & 54 & 52 & 54 & 54 \\
\hline \multirow[t]{3}{*}{21} & $\begin{array}{l}\text { Correlation } \\
\text { Coefficient }\end{array}$ & $.319^{*}$ & $.307^{*}$ & 1.000 & -.097 & $.381^{* *}$ & $.298^{*}$ & .126 & $.273^{*}$ & $.274^{*}$ & $.490^{* *}$ \\
\hline & Sig. (2-tailed) & .020 & .024 & & .452 & .002 & .019 & .329 & .045 & .031 & .000 \\
\hline & $\mathrm{N}$ & 53 & 54 & 62 & 62 & 62 & 62 & 62 & 54 & 62 & 62 \\
\hline \multirow[t]{3}{*}{22} & $\begin{array}{l}\text { Correlation } \\
\text { Coefficient }\end{array}$ & -.186 & -.166 & -.097 & 1.000 & -.128 & .173 & -.080 & .133 & .132 & -.070 \\
\hline & Sig. (2-tailed) & .181 & .230 & .452 & & .320 & .178 & .534 & .336 & .307 & .586 \\
\hline & $\mathrm{N}$ & 53 & 54 & 62 & 62 & 62 & 62 & 62 & 54 & 62 & 62 \\
\hline \multirow[t]{3}{*}{23} & $\begin{array}{l}\text { Correlation } \\
\text { Coefficient }\end{array}$ & $.433^{* *}$ & $.328^{*}$ & $.381^{* *}$ & -.128 & 1.000 & .137 & $.360^{* *}$ & $.272^{*}$ & .140 & $.337^{* *}$ \\
\hline & Sig. (2-tailed) & .001 & .015 & .002 & .320 & & .287 & .004 & .047 & .279 & .007 \\
\hline & $\mathrm{N}$ & 53 & 54 & 62 & 62 & 62 & 62 & 62 & 54 & 62 & 62 \\
\hline \multirow[t]{3}{*}{24} & $\begin{array}{l}\text { Correlation } \\
\text { Coefficient }\end{array}$ & $.279^{*}$ & .079 & $.298^{*}$ & .173 & .137 & 1.000 & .023 & $.455^{* *}$ & .112 & .185 \\
\hline & Sig. (2-tailed) & .043 & .572 & .019 & .178 & .287 & & .858 & .001 & .384 & .149 \\
\hline & $\mathrm{N}$ & 53 & 54 & 62 & 62 & 62 & 62 & 62 & 54 & 62 & 62 \\
\hline \multirow[t]{3}{*}{25} & $\begin{array}{l}\text { Correlation } \\
\text { Coefficient }\end{array}$ & .224 & $.306^{*}$ & .126 & -.080 & $.360^{* *}$ & .023 & 1.000 & .165 & .193 & $.300^{*}$ \\
\hline & Sig. (2-tailed) & .107 & .024 & .329 & .534 & .004 & .858 & & .235 & .133 & .018 \\
\hline & $\mathrm{N}$ & 53 & 54 & 62 & 62 & 62 & 62 & 62 & 54 & 62 & 62 \\
\hline \multirow[t]{3}{*}{26} & $\begin{array}{l}\text { Correlation } \\
\text { Coefficient }\end{array}$ & $.523^{* *}$ & $.472^{* *}$ & $.273^{*}$ & .133 & $.272^{*}$ & $.455^{* *}$ & .165 & 1.000 & $.379^{* *}$ & $.402^{* *}$ \\
\hline & Sig. (2-tailed) & .000 & .000 & .045 & .336 & .047 & .001 & .235 & & .005 & .003 \\
\hline & $\mathrm{N}$ & 52 & 52 & 54 & 54 & 54 & 54 & 54 & 54 & 54 & 54 \\
\hline
\end{tabular}




\begin{tabular}{|l|l|r|r|r|r|r|r|r|r|r|r|}
28 & $\begin{array}{l}\text { Correlation } \\
\text { Coefficient }\end{array}$ & .219 & .265 & $.274^{*}$ & .132 & .140 & .112 & .193 & $.379^{* *}$ & 1.000 & .249 \\
\cline { 2 - 11 } & Sig. (2-tailed) & .116 & .053 & .031 & .307 & .279 & .384 & .133 & .005 & & .051 \\
\cline { 2 - 12 } & $\mathrm{N}$ & 53 & 54 & 62 & 62 & 62 & 62 & 62 & 54 & 62 & 62 \\
\hline 33 & $\begin{array}{l}\text { Correlation } \\
\text { Coefficient }\end{array}$ & $.383^{* *}$ & $.485^{* *}$ & $.490^{* *}$ & -.070 & $.337^{* *}$ & .185 & $.300^{*}$ & $.402^{* *}$ & .249 & 1.000 \\
\cline { 2 - 11 } & Sig. (2-tailed) & .005 & .000 & .000 & .586 & .007 & .149 & .018 & .003 & .051 & \\
\cline { 2 - 11 } & $\mathrm{N}$ & 53 & 54 & 62 & 62 & 62 & 62 & 62 & 54 & 62 & 62 \\
\hline
\end{tabular}

*. Correlation is significant at the 0.05 level (2-tailed).

**. Correlation is significant at the 0.01 level (2-tailed). 